\title{
A versatile and modular tetrode-based device for single-unit recordings in rodent ex vivo and in vivo acute preparations
}

Francisca Machado ${ }^{1,2}$, Nuno Sousa ${ }^{1,2}$, Patricia Monteiro ${ }^{1,2^{*}}$, Luis Jacinto ${ }^{1,2^{\star}}$

1- Life and Health Sciences Research Institute (ICVS), School of Medicine, University of Minho, 4710-057 Braga, Portugal.

2- ICVS/3B's-PT Government Associate Laboratory, Braga/Guimarães, Portugal.

Corresponding authors:

*Luis Jacinto and *Patricia Monteiro

Email: luisjacinto@med.uminho.pt

Email: patriciamonteiro@med.uminho.pt

\section{Keywords}

Tetrodes; Extracellular Electrophysiology; Rodent recordings; Ex vivo brain slices; In vivo auditory cortex

\section{Author Contributions}

Francisca Machado: Investigation, Formal Analysis, Software, Data Curation, Writing - original draft preparation; Nuno Sousa: Conceptualization, Funding Acquisition; Patricia Monteiro: Conceptualization, Methodology, Validation, Supervision, Resources, Funding Acquisition, Writing - reviewing and editing; Luis Jacinto: Conceptualization, Methodology, Software, Validation, Supervision, Writing - reviewing and editing. 
bioRxiv preprint doi: https://doi org/10.1101/2020.02 11.940809. this version posted February 12, 2020. The copyright holder for this preprint (which was not certified by peer review) is the author/funder, who has granted bioRxiv a license to display the preprint in perpetuity. It is made available under aCC-BY-NC-ND 4.0 International license.

\begin{abstract}
The demand for affordable tools for recording extracellular activity and successfully isolating single units from different brain preparations has pushed researchers and companies to invest in developing and fabricating new recording devices. However, depending on the brain region of interest, experimental question or type of preparations, different devices are required thus adding substantial financial burden to laboratories. We have developed a simple and affordable tetrodebased device that allows interchangeable extracellular recordings of neural activity between in vivo and ex vivo preparations and can be easily implemented in all wet-bench laboratories. Spontaneous activity from several putative single neurons could be easily recorded and isolated by lowering the device into ex vivo cerebellum brain slices. The same device was also used in vivo, lowered into primary auditory cortex of adult anesthetized transgenic mice expressing channelrhodopsin in cortical neurons. Acoustic stimulation of the contralateral ear or direct laser optogenetic stimulation successfully evoked cortical activity at the recording site. Several isolated putative single neurons presented time-locked activity response to the different stimuli. In summary, we developed an affordable, versatile and modular device to facilitate tetrode extracellular recordings interchangeably between in vivo anaesthetized animals and ex vivo brain slice recordings.
\end{abstract}

\title{
Highlights
}

Developed a versatile and modular device to facilitate tetrode acute brain recordings interchangeably between in vivo and ex vivo preparations.

Conducted ex vivo extracellular recordings in acute cerebellar slices.

Conducted in vivo extracellular recordings in auditory cortex of anaesthetized mice.

Recorded and isolated multiple single units in both acute slices and anaesthetized mice recordings using the same device.

Device can be easily extended to accommodate optic fiber and cannula. 


\section{Introduction}

Extracellular neuronal activity has been recorded and analyzed in neuroscience research for decades, providing insight into cell- and circuit-level brain computations [1], [2]. The ability to perform spike-sorting on extracellular activity recordings has been particularly useful since it allows the isolation of putative single neurons and the analysis of their activity/responses individually [3]. However, spike sorting is less commonly used in ex vivo brain slice recordings because typical single-wire pipette extracellular recordings are not ideal for this purpose and commercially available options are usually costly, namely multi-electrode arrays (MEA) [4], [5] and especially designed silicon probes [6]. Tetrodes are a simple and cost-effective approach that has been routinely used for chronic recordings of extracellular activity in freely-moving rodents [7]. Their long lasting success in neuroscience relies not only on their cost and easy implementation but also on their ability to facilitate spike sorting [7], [8]. Surprisingly, despite their success in freely-moving electrophysiology, tetrodes have been less frequently used in acute preparations including in vivo anesthetized animals and especially ex vivo slice preparations. One main factor limiting the use of tetrodes for the recording of extracellular spikes and subsequent unit isolation in acute preparations is the non-existence of commercially available devices that can simultaneously work as tetrode interface boards and structurally support/guide tetrodes into slices or whole-brains in anaesthetized animals.

Here, we propose a versatile and modular tetrode-based device that takes inspiration from probe design and freely-moving rodent tetrode microdrives, working for both ex vivo brain slices and in vivo anaesthetized recordings. The device parts can be easily produced by affordable and widespread technologies, many times in-lab, such as printed circuit board (PCB) manufacturing and $3 \mathrm{D}$ printing. Additionally, the device can be: quickly assembled; integrated with any amplifier/recording system already available at the lab for extracellular recordings and; used unlimited times, including inter-changeably between ex vivo and in vivo experiments, with multiple tetrodes and configurations. For in vivo preparations it can also be easily extended to be coupled with optic fiber or cannulas for optogenetic and pharmacological experiments. Using this device, we recorded spontaneous and opto- and sound- evoked neuronal activity in transgenic mice, both in vivo and ex vivo. In both configurations it was possible to reliably isolate several good quality single units from short recordings and analyze individual units' responses to light and sound modulation. This device will facilitate a more widespread use of tetrodes for acute preparations in neuroscience experiments.

\section{Materials and methods}

\subsection{Tetrode fabrication}

Nichrome tetrodes (NiCr,12.5 $\mu$ m diameter, RO-800 Hard PAC, Sandvik) were fabricated with standard methods, described elsewhere [9]. Briefly, a $50 \mathrm{~cm}$ long strand of insulated $\mathrm{NiCr}$ wire was folded and twisted by a motorized tetrode spinner (Tetrode Spinner 2.0, Neuralynx) while being tensioned. After the twisting procedure, the wires' insulation was fused at $420^{\circ} \mathrm{C}$ with the 
bioRxiv preprint doi: https://doi.org/10.1101/2020.02.11.940809; this version posted February 12, 2020. The copyright holder for this preprint (which was not certified by peer review) is the author/funder, who has granted bioRxiv a license to display the preprint in perpetuity. It is made available under aCC-BY-NC-ND 4.0 International license.

aid of a heat gun and then carefully cut at the top and bottom. Tetrodes were then stored for later use.

\subsection{Electrode interface board and tetrode guide structure}

The proposed device consists of two parts: an electrode interface board (EIB) and a tetrode guide structure (Figure 1).

The EIB is a printed circuit board (PCB) that allows the electrical connection of tetrode wires to gold coated vias $(0.3 \mathrm{~mm}$ diameter) in the board by means of gold pins (small EIB pins, Neuralynx). These vias are routed to a female omnetics connector (A79024-001, Omnetics Connector Corporation) that mates with an appropriate headstage with male omnetics connector (which are used by many electrophysiology companies including Open Ephys, Neuralynx and Plexon, for example). There are 32 electrode vias (for 8 tetrodes) plus 2 ground and 2 reference vias. The 2 ground vias are shorted together. The EIB is a 2-layer PCB (10 mil trace/space, 1.6 $\mathrm{mm}$ thickness) and was manufactured with electroless nickel immersion gold (ENIG) surface finishing. The omnetics connector was soldered to the board by hot air soldering with solder paste (Leaded, No Clean Solder Paste, MG Chemicals). A $40 \mathrm{~cm}$ long 4-gauge isolated copper wire was looped through two drill holes on the PCB, designed for the purpose, and soldered to the ground vias.

The tetrode guide structure physically holds and guides the tetrodes into the tissue. This structure was 3D-printed in plastic (PA12 multijet fusion plastic; $70 \times 10 \times 1.5 \mathrm{~mm}$ ) and has a single groove track that, depending on dimensions, can hold up to 8 tightly packed tetrodes lined in a single row. The support structure can be printed in any plastic material by any 3D printer with sufficient precision to print the tetrode guide track. The number, size and position of the tracks can also be easily adjusted by simple changes to the 3D part design file. The EIB and the tetrode guide structure were connected together by means of two $2 \mathrm{~mm}$ diameter screws. There are two square holes on both parts that line up and allow passing of tetrode wires from one side of the board to the other, if necessary.

Tetrodes were placed in the guide track with the aid of ceramic coated tweezers (1125250, Fine Science Tools) under a microscope (SZ51, Olympus) and glued in place with 5-minute epoxy (Devcon). Each tetrode wire was then routed to an electrode via in the EIB and secured with a gold pin. Tightly pressing the gold pin against the electrode wire inside the via, removes the electrode's insulation and allows electrical connection. Figure 1B shows a fully assembled device with $8 \mathrm{NiCr}$ tetrodes loaded and connected to the EIB.

\subsection{Tetrode electrodeposition}

After gluing the tetrodes to the guide structure and physically connecting each tetrode wire to the electrode vias in the EIB, tetrodes' tips were cut with sharp stainless-steel scissors (14568-09, Fine Science Tools) and electroplated. The plating solution consisted of $5 \%$ gold non-cyanide solution (Sifco 5355 Gold Plating Solution, Neuralynx) with 1\% Poly(ethylene glycol) (PEG) addictive (BioUltra, 8,000, Sigma-Aldrich) mixed in a 75\% v/v PEG to gold ratio. This solution has been shown to promote a large surface area with low impedance [10]. Electrode impedance was lowered to $150-200 \mathrm{KOhm}$ in successive steps of electrodeposition at $-0.05 \mu \mathrm{A}$ with the aid of NanoZ (White Matter LLC). 
bioRxiv preprint doi: https://doi.org/10.1101/2020.02.11.940809; this version posted February 12, 2020. The copyright holder for this preprint (which was not certified by peer review) is the author/funder, who has granted bioRxiv a license to display the preprint in perpetuity. It is made available under aCC-BY-NC-ND 4.0 International license.

\subsection{Ex-vivo and in-vivo electrophysiological recordings}

\subsubsection{Animals}

Emx1-Cre:Ai27D or Pvalb-Cre:Ai27D male mice were originally purchase from JAX (stocks \#008069, \#005628, \#012567) [11]-[13]. All experiments were conducted in accordance with European Union Directive 2016/63/EU and the Portuguese regulations and laws on the protection of animals used for scientific purposes (DL No 113/2013). This study was approved by the Ethics Subcommittee for the Life Sciences and Health of University of Minho (ID: SECVS 01/18) and the Portuguese Veterinary General Direction (ID: DGAV 8519).

\subsubsection{Ex vivo cerebellar electrophysiological recordings}

\subsubsection{Ex vivo slice preparation and recordings}

Briefly, animals were deeply anaesthetized with avertin $(0.5 \mathrm{mg} / \mathrm{g}$ body weight; tribromoethanol; $20 \mathrm{mg} / \mathrm{mL}$; Sigma-Aldrich) by intraperitoneal injection and decapitated. The brain was quickly removed to prepare $300 \mu \mathrm{m}$ cerebellum parasagittal slices according to the protocol of [14]. All recordings were performed at $34^{\circ} \mathrm{C}$ in artificial cerebrospinal fluid gassed with $5 \% \mathrm{CO}_{2} / 95 \% \mathrm{O}_{2}$. The tetrode device containing $8 \mathrm{NiCr}$ electroplated tetrodes was secured to a 3-axis micromanipulator (PatchStar, Scientifica) and connected to a 32-channel headstage (RHD2132, Intan). The tetrodes were then guided into the slice with the micromanipulator under visual inspection of a microscope (Figure 1C) and placed close to the Purkinje cell layer at cerebellum lobule IV. Extracellular activity was recorded with an Open Ephys acquisition system (Open Ephys) [15] connected to the headstage by serial peripheral interface (SPI) cables (Intan). Signals were acquired at $30 \mathrm{Ks} / \mathrm{s}$.

\subsubsection{In vivo auditory cortex electrophysiological recordings}

\subsubsection{Surgical procedure and in vivo extracellular recordings}

Mice were anesthetized by intraperitoneal injection mix of ketamine $(75 \mathrm{mg} / \mathrm{Kg})$ and medetomidine $(1 \mathrm{mg} / \mathrm{Kg})$ and positioned in a stereotaxic frame (Stoelting). Dorsal skull was exposed and the animal was rotated $90^{\circ}$ degrees to facilitate removal of the temporal muscle and access to the primary auditory cortex (A1). A $1.5 \times 1.5 \mathrm{~mm}$ area above A1 centered at $-2.5 \mathrm{~mm}$ AP and $4.0 \mathrm{~mm}$ $M L$ from bregma [16] was opened and the dura removed under a microscope (S6, Leica Mycrosystems). The tetrode device containing $8 \mathrm{NiCr}$ electroplated tetrodes was attached to a micrometric stereotaxic arm (1760, Kopf Instruments) and connected to an headstage (RHD2132, Intan). Tetrodes were lowered into the brain, through the skull opening, to $-0.6 \mathrm{~mm}$ (DV) from the brain surface. A stainless-steel screw electrode (E363-20-2.4-03, Plastics One) secured in a burr hole at the back of the skull served as ground. The surgical procedure and the following stimulation experiments were conducted on a custom-made anti-vibration table inside a doublewalled sound-proof chamber. Extracellular signals were acquired with an Open Ephys acquisition system at $30 \mathrm{Ks} / \mathrm{s}$.

\subsubsection{Evoked auditory response}

To evoke responses from A1 neurons, tones of variable frequency $(8,12,20,24,28$ and $32 \mathrm{KHz}$, 25 ms duration, $975 \mathrm{~ms}$ intertrial interval) and variable sound pressure level $(50,60,70$ and 80 $\mathrm{dB}$ SPL) were pseudo-randomly delivered from a free field electrostatic speaker (ES1, TDT) positioned $10 \mathrm{~cm}$ away from the mouse's contralateral ear. The speaker was driven by a speaker 
bioRxiv preprint doi: https://doi.org/10.1101/2020.02.11.940809; this version posted February 12, 2020. The copyright holder for this preprint (which was not certified by peer review) is the author/funder, who has granted bioRxiv a license to display the preprint in perpetuity. It is made available under aCC-BY-NC-ND 4.0 International license.

driver (ED1, Tucker-Davis Technologies) and tones were generated by an high-sampling rate sound card (HARP, Champalimaud Foundation) controlled by Bonsai [17].

\subsubsection{Optogenetic stimulation}

To evoke neuronal activity of A1 neurons, the surface of the exposed brain was illuminated with blue light pulses ( $473 \mathrm{~nm}$ wavelength, $10 \mathrm{~Hz}$ with $30 \mathrm{~ms}$ pulse width, $5 \mathrm{~s}$ On, 55s Off). Light was delivered by an optical fiber (200 $\mu \mathrm{m}$ diameter) connected to a fiber-coupled DPSS laser source (CNI). Light pulses were generated by a waveform function generator (DG1022, Rigol) connected to the laser source. The optical fiber was held by 3-axis micromanipulator (LBM-2025-00, Scientifica) and placed $5 \mathrm{~mm}$ above brain surface.

\subsubsection{Signal processing and spike sorting}

Signals were analyzed with custom-written Matlab (Mathworks) code. Both ex vivo and in vivo recordings were filtered between 0.6 and $6 \mathrm{KHz}$. Spikes were detected using a variable amplitude threshold that was a multiple (4 to 6 times) of the median from an estimate of the background noise's standard deviation as in [18]. Spike sorting was achieved by performing weighted principal component analysis (wPCA) on the waveforms of detected spikes followed by Gaussian Mixture Model (GMM) unsupervised clustering of the first principal components of pairs of electrodes from the same tetrode (Figure 2B) [19]. In a final step, clusters were manually split or merged based on visual inspection of cluster waveforms and their inter-spike interval histograms and autocorrelograms.

\section{Results}

The device was assembled and ready for recordings in under 2 hours including 1 hour for fabrication of eight tetrodes and 1 hour for assembly, tetrode loading and electroplating. The same tetrode tips were cut fresh and electroplated between the ex vivo and in vivo experiments.

In the ex vivo brain slice experiment (Figure 2A-C) we were able to isolate nine putative single neurons from eight tetrodes positioned at the Purkinje cell layer (cerebellum lobule IV) during a 30 minute recording of spontaneous activity. Figure $2 \mathrm{C}$ shows 4 units isolated from a single tetrode wire.

In the in vivo experiment (Figure 2D-H) several putative neurons were isolated from six tetrodes during a 30 minute recording from the primary auditory cortex in an anesthetized mouse. Additionally, by identifying putative neurons through spike sorting, we were able to analyze singleunits' responses to specific light and sound stimuli (Figure $2 \mathrm{~F}-\mathrm{H}$ ).

\section{Discussion}

This manuscript describes the design and implementation of an affordable, versatile and modular tetrode-based device that allows extracellular recordings in both ex vivo and in vivo preparations. The same device can be used in both preparations without any changes in configuration or parts, as performed here. Its probe-like design allows easy positioning in micromanipulators for ex vivo slice recordings and in stereotaxic frames for in vivo recordings. The proposed device is solely based on parts that can be easily produced in-lab or sourced for a low cost. This is especially 
bioRxiv preprint doi: https://doi.org/10.1101/2020.02.11.940809; this version posted February 12, 2020. The copyright holder for this preprint (which was not certified by peer review) is the author/funder, who has granted bioRxiv a license to display the preprint in perpetuity. It is made available under aCC-BY-NC-ND 4.0 International license.

important considering the cost of MEAs and silicon probes, which might be prohibitive for many labs especially those running experiments demanding constant re-use.

The EIB was inspired by EIBs from microdrive systems for tetrode positioning in freelymoving animals [20]-[22] where the easiness of assembly must be combined with quick and nondamaging part recovery. PCB manufacturing is currently a widespread service accessible at a low cost. The present EIB was designed to match headstages with male omnetics connectors which are used by many traditional electrophysiology systems such as Open Ephys (used in the experiments described here), Neuralynx and Plexon; but can be easily changed and produced to accommodate other types of connectors for other headstages.

Similarly, 3D printing has also become an affordable technology and most labs now have access to it in-house. The tetrode guide structure design was inspired by silicon probe tip's design and can be printed in virtually any commercial or in-house 3D printer, contingent to printer's precision and the desired guide track's dimensions. Since 3D printing allows easy customization, the proposed design for the guide structure can be easily changed by simple alterations to the 3D part design file to accommodate several configurations, from single tetrode tracks to multiple tracks in the same or different locations. The possibility of changing the track dimensions and position allows versatile tetrode positioning - for example, for targeting different brain structures simultaneously - and the use of tetrode wires of different diameters for different experimental demands. The guide design presented here can also be modified to accommodate optic fiber tracks for optogenetic experiments or cannula for pharmacological experiments. Despite this versatility, and although tetrodes can be cut with precision scissors and lined up in a row with micrometric precision under a microscope, when compared with MEAs and silicon probes, their real geometry is not fixed/known which requires, for example, additional modeling for current source density analysis studies [23].

The whole device was designed for constant re-use with minimal cost and minimal damage to the parts upon recovery. In fact, the same tetrodes glued to the guide structure and connected to the EIB can be used for several sequential recordings across different days as long as tetrode tips are cut fresh and electroplated between experiments (and as long as the tetrodes are of sufficient length for the intended target). Nevertheless, the whole device can be quickly recovered for a new experiment. The EIB can be re-used by removing the gold pins from the vias and discarding the tetrodes. The same gold pins can be re-used for the next application. The tetrode guide can be recovered by immersion in $70 \%$ alcohol solution overnight to remove the epoxy, or it can also be discarded between experiments given that it can be printed effortlessly.

The affordability and easiness of manufacturing and assembly of the proposed device will allow a more widespread use of tetrodes for acute extracellular recordings and unit isolation in ex vivo and in vivo preparations.

\section{Conflicts of interest}

None.

\section{Acknowledgements}

The authors would like to acknowledge Diana Rodrigues for helping preparing ex vivo brain slices and Margarida Gonçalves for helping with in vivo A1 surgeries. This work was supported by Calouste Gulbenkian Foundation (grant number P-139977); Society in Science, The Branco Weiss fellowship, administered by Eidgenössische Technische Hochschule (ETH) Zürich; the 
bioRxiv preprint doi: https://doi.org/10.1101/2020.02.11.940809; this version posted February $12,2020$. The copyright holder for this preprint (which was not certified by peer review) is the author/funder, who has granted bioRxiv a license to display the preprint in perpetuity. It is made available under aCC-BY-NC-ND 4.0 International license.

European Molecular Biology Organization (EMBO) Long-Term Fellowship (ALTF 89-2016 to P.M.) and FCT (grant number PTDC/MED-NEU/28073/2017, POCI-01-0145-FEDER-028073). This work was also funded by FEDER through the Competitiveness Factors Operational Programme (COMPETE), by National funds through the Foundation for Science and Technology (FCT) under the scope of the project UID/Multi/50026; and by the project NORTE-01-0145FEDER-000013, supported by the Northern Portugal Regional Operational Programme (NORTE 2020), under the Portugal 2020 Partnership Agreement, through the European Regional Development Fund (FEDER).

\section{References}

[1] D. A. Henze, Z. Borhegyi, J. Csicsvari, A. Mamiya, K. D. Harris, and G. Buzsáki, "Intracellular features predicted by extracellular recordings in the hippocampus in vivo," $J$. Neurophysiol., 2000, doi: 10.1152/jn.2000.84.1.390.

[2] P. Barthó, H. Hirase, L. Monconduit, M. Zugaro, K. D. Harris, and G. Buzsáki, "Characterization of neocortical principal cells and interneurons by network interactions and extracellular features," J. Neurophysiol., 2004, doi: 10.1152/jn.01170.2003.

[3] H. G. Rey, C. Pedreira, and R. Quian Quiroga, "Past, present and future of spike sorting techniques," Brain Research Bulletin. 2015, doi: 10.1016/j.brainresbull.2015.04.007.

[4] M. Meister, R. O. L. Wong, D. A. Baylor, and C. J. Shatz, "Synchronous bursts of action potentials in ganglion cells of the developing mammalian retina," Science (80-. )., 1991, doi: $10.1126 /$ science.2035024.

[5] M. O. Heuschkel, M. Fejtl, M. Raggenbass, D. Bertrand, and P. Renaud, "A threedimensional multi-electrode array for multi-site stimulation and recording in acute brain slices," J. Neurosci. Methods, 2002, doi: 10.1016/S0165-0270(01)00514-3.

[6] P. Aivar, M. Valero, E. Bellistri, and L. M. de la Prida, "Extracellular calcium controls the expression of two different forms of ripple-like hippocampal oscillations," J. Neurosci., 2014, doi: 10.1523/JNEUROSCI.2826-13.2014.

[7] M. A. Wilson and B. L. McNaughton, "Dynamics of the hippocampal ensemble code for space," Science (80-. )., 1993, doi: 10.1126/science.8351520.

[8] C. M. Gray, P. E. Maldonado, M. Wilson, and B. McNaughton, "Tetrodes markedly improve the reliability and yield of multiple single-unit isolation from multi-unit recordings in cat striate cortex," J. Neurosci. Methods, 1995, doi: 10.1016/0165-0270(95)00085-2.

[9] D. P. Nguyen et al., "Micro-drive array for chronic in vivo recording: Tetrode assembly," J. Vis. Exp., 2009, doi: 10.3791/1098.

[10] J. E. Ferguson, C. Boldt, and A. D. Redish, "Creating low-impedance tetrodes by electroplating with additives," Sensors Actuators, A Phys., 2009, doi: 10.1016/j.sna.2009.10.001.

[11] J. A. Gorski, T. Talley, M. Qiu, L. Puelles, J. L. R. Rubenstein, and K. R. Jones, "Cortical excitatory neurons and glia, but not GABAergic neurons, are produced in the Emx1expressing lineage," J. Neurosci., 2002, doi: 10.1523/jneurosci.22-15-06309.2002.

[12] S. Hippenmeyer et al., "A developmental switch in the response of DRG neurons to ETS transcription factor signaling," PLOS Biol., 2005, doi: 10.1371/journal.pbio.0030159.

[13] L. Madisen et al., "A toolbox of Cre-dependent optogenetic transgenic mice for lightinduced activation and silencing," Nat. Neurosci., 2012, doi: 10.1038/nn.3078. 
[14] S. Huang and M. Y. Uusisaari, "Physiological temperature during brain slicing enhances the quality of acute slice preparations," Front. Cell. Neurosci., 2013, doi: 10.3389/fncel.2013.00048.

[15] J. H. Siegle, A. C. López, Y. A. Patel, K. Abramov, S. Ohayon, and J. Voigts, "Open Ephys: An open-source, plugin-based platform for multichannel electrophysiology," J. Neural Eng., 2017, doi: 10.1088/1741-2552/aa5eea.

[16] K. B. J. Franklin and G. Paxinos, The Mouse Brain in Stereotaxic Coordinates (map). 2007.

[17] G. Lopes et al., "Bonsai: An event-based framework for processing and controlling data streams," Front. Neuroinform., 2015, doi: 10.3389/fninf.2015.00007.

[18] R. Q. Quiroga, Z. Nadasdy, and Y. Ben-Shaul, "Unsupervised spike detection and sorting with wavelets and superparamagnetic clustering," Neural Comput., 2004, doi: $10.1162 / 089976604774201631$.

[19] B. C. Souza, V. Lopes-dos-Santos, J. Bacelo, and A. B. L. Tort, "Spike sorting with Gaussian mixture models," Sci. Rep., 2019, doi: 10.1038/s41598-019-39986-6.

[20] J. Voigts, J. Siegle, D. L. Pritchett, and C. I. Moore, "The flexDrive: An ultra-light implant for optical control and highly parallel chronic recording of neuronal ensembles in freely moving mice," Front. Syst. Neurosci., 2013, doi: 10.3389/fnsys.2013.00008.

[21] F. Kloosterman et al., "Micro-drive array for chronic in vivo recording: Drive fabrication," $J$. Vis. Exp., 2009, doi: 10.3791/1094.

[22] E. H. Chang, S. A. Frattini, S. Robbiati, and P. T. Huerta, "Construction of microdrive arrays for chronic neural recordings in awake behaving mice.," J. Vis. Exp., 2013, doi: $10.3791 / 50470$.

[23] K. H. Pettersen, H. Lindén, A. M. Dale, and G. T. Einevoll, "Extracellular spikes and current-source density," Handb. neural Act. Meas. Cambridge Univ. Press. Cambridge, UK, to Appear, 2010. 
bioRxiv preprint doi: https://doi org/10.1101/2020.02 11 940809; this version posted February 12, 2020. The copyright holder for this preprint (which was not certified by peer review) is the author/funder, who has granted bioRxiv a license to display the preprint in perpetuity. It is made available under aCC-BY-NC-ND 4.0 International license.

3D Device design

A)
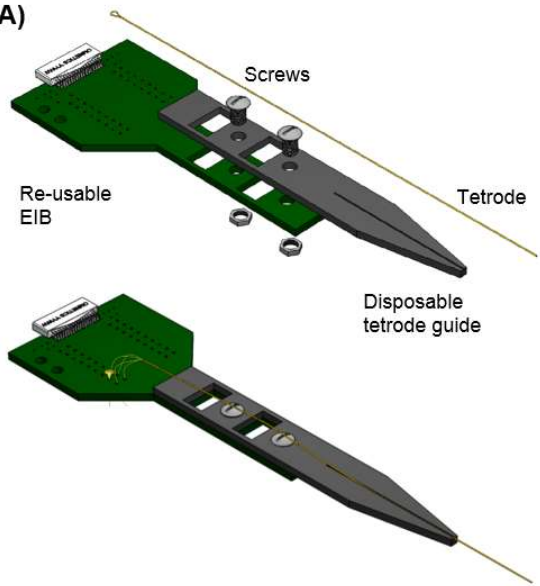

Assembled device

B)

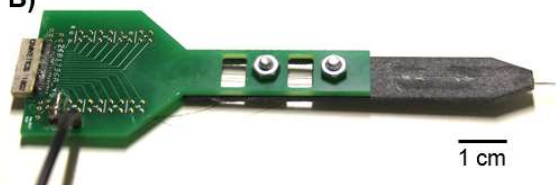

C)

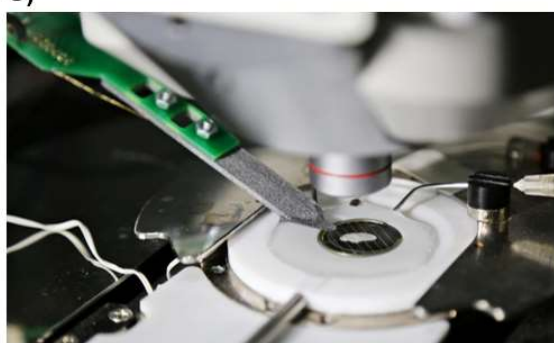

Figure 1 - Tetrode device design overview. (A) 3D rendering of the device's parts, including the electrode interface board (EIB) and the tetrode guide, separately (top) and fully assembled (bottom). (B) Photo of assembled device with loaded NiCr tetrodes and ground wire. (C) Ex vivo recording with device being held by micromanipulator and tetrodes guided into the slice inside the recording chamber. 

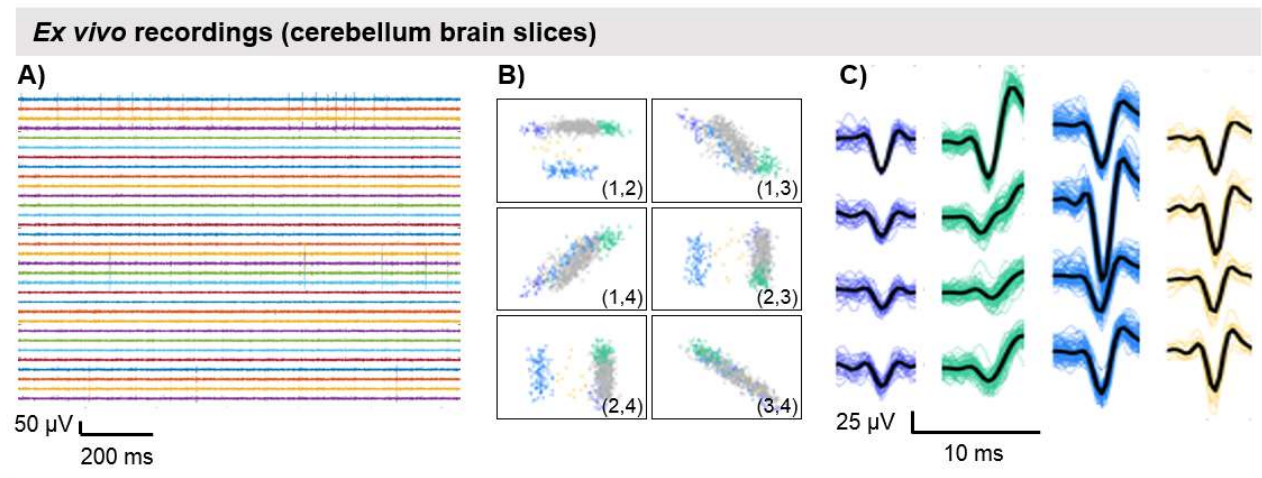

In vivo anesthetized recordings

D) Primary auditory cortex (A1) F) Optogenetic stimulation

G) Sound stimulation ( $8 \mathrm{kHz}, 80 \mathrm{~dB})$
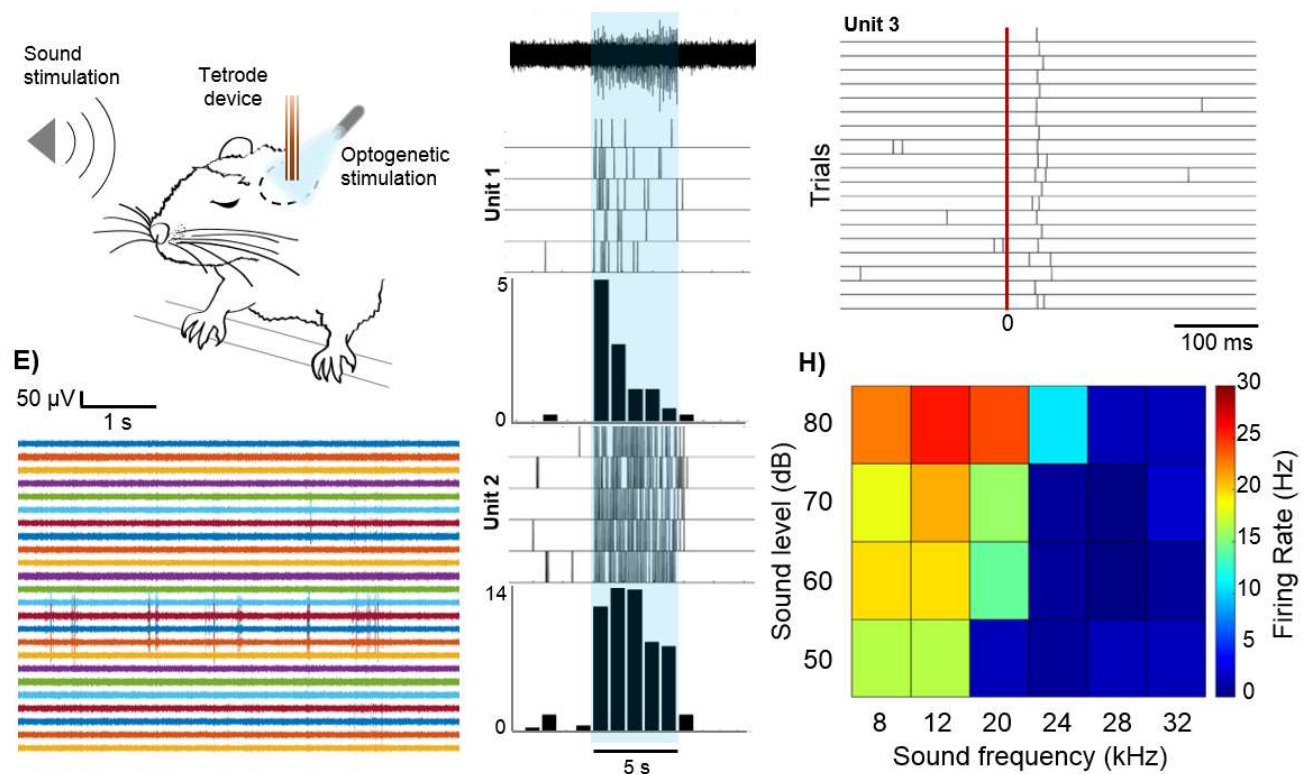

Figure 2 - Ex vivo and in vivo electrophysiological recordings. (A) Extracellular signals recorded on 8 tetrodes from a mouse cerebellum brain slice (signals band-passed filtered between 0.6 and $6 \mathrm{KHz}$ ). (B) Cluster isolation for one tetrode using PCA and GMM based spike sorting. Each scatterplot shows the projections of two pairs of tetrode's channel waveforms onto the first principal component. Colored points correspond to isolated single units (matching colors with respective waveforms on right). Grey points correspond to multi-unit activity which was not possible to isolate into single units. (C) Sample waveforms of four isolated single units from one tetrode (average waveform in black). (D) Representative drawing of the in vivo experiment combining tetrode recordings with optogenetic and sound stimulation in primary auditory cortex (A1) of an anesthetized mouse. (E) Extracellular signals recorded on 6 tetrodes from A1 (signals band-passed filtered between 0.6 and $6 \mathrm{KHz}$ ). (F) Evoked activity of isolated single units in $\mathrm{A} 1$ by optogenetic stimulation. Extracellular activity recorded during one trial of light stimulation (light stimulation period in blue, corresponding to 5 seconds) (top); Raster plots and average peristimulus time histograms of two isolated single units over five trials. (G-H) Evoked activity of one isolated single unit in A1 by sound stimulation. (G) Raster plot of one isolated single unit responding to $8 \mathrm{KHz}$ tone stimuli over 20 presentations (red line marks tone presentation). $(\mathrm{H})$ Tuning heatmap of the same unit showing average firing rate across the different sound frequencies and sound levels presented during the evoked auditory response protocol. 